\title{
Synthesis and biological evaluation of novel 1,3,4-thiadiazole derivatives as possible anticancer agents
}

\author{
ULVIYE ACAR ÇEVIK ${ }^{1,2 *}$ \\ DERYA OSMANIYE1,2 \\ SERKAN LEVENT T,2 \\ BEGÜM NURPELIN SAĞLIK ${ }^{1,2}$ \\ BETÜL KAYA ÇAVUŞOĞLU1,2 \\ ABDULLAH BURAK KARADUMAN ${ }^{3}$ \\ YUSUF ÖZKAY ${ }^{1,2}$ \\ ZAFER ASIM KAPLANCIKLI ${ }^{1}$ \\ ${ }^{1}$ Department of Pharmaceutical \\ Chemistry, Faculty of Pharmacy \\ Anadolu University, 26470 Eskişehir \\ Turkey \\ ${ }^{2}$ Doping and Narcotic Compounds \\ Analysis Laboratory, Faculty \\ of Pharmacy, Anadolu University \\ 26470 Eskişehir, Turkey \\ ${ }^{3}$ Department of Pharmaceutical \\ Toxicology, Faculty of Pharmacy \\ Anadolu University, 26470 Eskişehir \\ Turkey \\ Accepted November 19, 2019 \\ Published online December 16, 2019
}

\begin{abstract}
The synthesis of new N-(5-substituted-1,3,4-thiadiazol-2-yl)-2-[(5-(substituted amino)-1,3,4-thiadiazol-2-yl)thio]acetamide derivatives and investigation of their anticancer activities were the aims of this work. All the new compounds' structures were elucidated by elemental analyses, IR, ${ }^{1} \mathrm{H}$ NMR, ${ }^{13} \mathrm{C}$ NMR and MS spectral data. Anticancer activity studies of the compounds were evaluated against MCF-7 and A549 tumor cell lines. In addition, with the purpose of determining the selectivity of cytotoxic activities, the most active compound was screened against a noncancer NIH3T3 cell line (mouse embryonic fibroblast cells). Among the tested compounds, compound $4 \mathbf{y}$ ( $N$-(5-ethyl-1,3,4-thiadiazol-2-yl)-2-((5-( $p$-tolylamino)-1,3,4-thiadiazol-2-yl)thio)acetamide), showed promising cytotoxic activity against MCF7 cancer cell with an $I C_{50}$ value of $0.084 \pm 0.020 \mathrm{mmol} \mathrm{L}^{-1}$ and against A549 cancer cell with $I C_{50}$ value of $0.034 \pm 0.008 \mathrm{mmol} \mathrm{L}^{-1}$, compared with cisplatin. The aromatase inhibitory activity was evaluated for compound 4y on MCF-7 cell line showing promising activity with $I C_{50}$ of $0.062 \pm 0.004 \mathrm{mmol} \mathrm{L}^{-1}$.
\end{abstract}

Keywords: 1,3,4-thiadiazole, anticancer activity, aromatase inhibitory activity, MTT assay

Cancer remains a major cause of death worldwide despite multiple approaches used in prevention and therapy. After lung cancer, breast cancer is the second leading cause of cancer death among women. About one million women in the world are diagnosed with breast cancer every year. Moreover, according to World Conference on Breast Cancer, around 400,000 women die from this disease each year $(1,2)$.

The investigations for breast cancer suggest that many tumors occur and develop through estrogen-dependent mechanisms (3). Accordingly, it can be assumed that estrogen deprivation may prevent the formation of these cancers or may result in the regression of occurred tumors. The target of this suppression is the inhibition of the aromatase enzyme, responsible for the cyclization and structuring of estrogen (4).

\footnotetext{
*Correspondence, e-mail: uacar@anadolu.edu.tr
} 


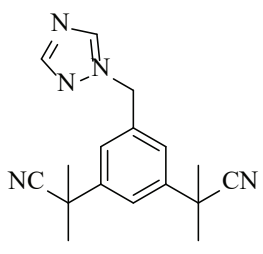

Anastrozole<smiles>N#Cc1ccc(C(c2ccc(C#N)cc2)n2cncn2)cc1</smiles>

Letrozole<smiles>Cn1nnc2ccc(C(c3ccc(Cl)cc3)n3cncn3)cc21</smiles>

Vorozole

Fig. 1. The third generation of aromatase inhibitors.

Aromatase is a very promising target for the endocrine treatment of estrogen-dependent diseases. In this sense, attention has been focused on the discovery of aromatase inhibitors able to control the progression of hormone-sensitive breast cancer in women, and decrease the circulating levels of estrogens $(5,6)$. According to their chronological order of clinical development, these inhibitors can be divided into three classes. The third-generation aromatase inhibitors (non-steroid aromatase inhibitors) are used as first-line therapy for the treatment of breast cancer in both early and advanced tumors as shown in Fig. 1. They are more selective and/or potent compared with first- and second-generation agents (7).

The strongest interaction with aromatase is a coordinative bond between the lone electron pair of an aromatic heterocyclic nitrogen atom and the heme iron of the enzyme (8). Compounds containing heterocycles and more than one nitrogen also exert inhibitory activity towards aromatase (9).

Thiadiazole has been studied extensively for more than one hundred years due to its outstanding therapeutic applications. Thiadiazole is a 5-membered planar aromatic motif comprising a sulfur atom, which improves the liposolubility of thiadiazole derivatives and hence their pharmacokinetics (10). From the literature survey, it was noticed that 1,3,4-thiadiazole derivatives possess many pharmacological activities, such as antiviral (11), antibacterial (12), antileishmanial (13), antitubercular (14), antimycobacterial (15), anticonvulsant (16) and analgesic activity (17). Different mechanisms of the antitumor activity of 1,3,4-thiadiazoles have been reported, including specific inhibition of epidermal growth factor receptor (EGFR) kinase (18), carbonic anhydrase (19), phosphodiesterase-7 (PDE7) (20), tyrosine kinase (21) and aromatase (22). In the view of the aforesaid, we designed and synthesized two thiadiazole-ring systems.

\section{EXPERIMENTAL}

\section{Materials and methods}

All of the chemicals used in the study were purchased either from Merck (Merck KGaA, Germany) or Sigma-Aldrich (USA) and used without further purification.

The purity of the compounds was checked by classical TLC on silica gel 60 F254 (Merck).

Melting points of the synthesized compounds were determined using an MP90 series automatic melting point determination system (Mettler-Toledo, USA) and were presented as uncorrected.

${ }^{1} \mathrm{H}$ and ${ }^{13} \mathrm{C}$ NMR spectra were recorded in DMSO- $d_{6}$ by a Bruker digital FT-NMR spectrometer (Bruker Bioscience, USA) at $300 \mathrm{MHz}$ and $75 \mathrm{MHz}$, resp. (splitting patterns in 
the NMR spectra were designated as follows: $\mathrm{s}$ - singlet, $\mathrm{d}$ - doublet, $\mathrm{t}$ - triplet, $\mathrm{m}$ - multiplet; coupling constants $(J)$ were reported in hertz). $M+1$ peaks were determined by the Shimadzu LC/MS ITTOF system (Shimadzu, Japan).

\section{Syntheses}

General procedure for N-substituted-hydrazinecarbothioamides (1a-j). - A mixture of suitable isothiocyanate derivative $(0.02 \mathrm{~mol})$ and hydrazine hydrate $(0.04 \mathrm{~mol})$ was stirred in ethanol $(99 \%)(30 \mathrm{~mL})$ for $4 \mathrm{~h}$ at $80^{\circ} \mathrm{C}$. After completion of the reaction, the precipitated product was filtered and washed with cold-ethanol.

General procedure for 5-(substituted-amino)-1,3,4-thiadiazole-2-thiols (2a-j). - Carbon disulfide $(0.019 \mathrm{~mol})$ was added into a solution of compound $1 \mathrm{a}-\mathrm{j}(0.018 \mathrm{~mol})$ in EtOH $(99$ $\%)$ in the presence of sodium hydroxide $(0.019 \mathrm{~mol})$ and then the mixture was refluxed for $8 \mathrm{~h}$. Afterward, the solution was cooled and acidified to $\mathrm{pH} 4-5$ with hydrochloric acid and crystallized from ethanol.

General procedure for 2-chloro-N-(5-substituted-1,3,4-thiadiazol-2-yl)acetamides $(3 \boldsymbol{a}, \boldsymbol{b})$. - A solution of 5-(substituted-amino)-1,3,4-thiadiazole-2-thiols $(\mathbf{2 a - j}) \quad(0.06 \mathrm{~mol})$ in tetrahydrofuran was cooled in an ice bath in the presence of triethylamine $(0.07 \mathrm{~mol}, 10.2$ $\mathrm{mL})$, and chloroacetyl chloride $(0.07 \mathrm{~mol}, 5.8 \mathrm{~mL})$ was added dropwise with stirring. After completion of the reaction, the solvent was evaporated under reduced pressure, the product was washed with water, dried and recrystallized from ethanol.

General procedure for N-(5-substituted-1,3,4-thiadiazol-2-yl)-2-[(5-(substituted-amino)-1,3,4-thiadiazol-2-yl)thio]acetamides $(4 \boldsymbol{a}-\mathbf{y})$. In order to obtained the target compounds, compounds $3 \mathbf{a}, \mathbf{b}(2.5 \mathrm{mmol})$ and compounds $2 \mathbf{a}-\mathbf{j}(2.5 \mathrm{mmol})$ were reacted at room temperature in acetone. After completion of the reaction, the solvent was evaporated under reduced pressure, product was washed with water, dried and recrystallized from ethanol.

The synthesis pathway of the target compounds $4 \mathbf{a}-\mathbf{y}$ is given in Scheme 1.

Physicochemical data of compounds $4 \mathbf{a}-\mathbf{y}$ are given in Table I. The final compounds were purified and their structures were characterized by spectroscopic methods (FT-IR, ${ }^{1} \mathrm{H}$ NMR, ${ }^{13} \mathrm{C}$ NMR, mass spectroscopy and elemental analyses). Spectral data of compounds $4 \mathbf{a}-\mathbf{y}$ are given in Table II.

\section{Cytotoxicity test}

MTT assay, based on the ability of metabolically active cells to convert the pale yellow MTT to a spectrophotometrically measured blue formazan salt, is one of the most favoured cytotoxicity tests (23). All of the synthesized compounds at various concentrations $(1,0.316$, $0.1,0.0316,0.01,0.00316,0.001$ and $\left.0.000316 \mathrm{mmol} \mathrm{L}^{-1}\right)$ were assayed for anticancer activity in cancer cell lines A549 and MCF-7 (ATCC, USA) using MTT method (24, 25). Cisplatin (Sigma Aldrich, USA) was used as a reference drug. The cytotoxic properties of the most active compounds (4j, $\mathbf{4} \mathbf{v}$ and $4 \mathbf{y})$ were evaluated using NIH3T3 cell line (ATCC) $(23,24)$.

\section{Aromatase inhibition assay}

This assay was carried out according to the kit procedure [Aromatase (CYP19A) Inhibitor screening kit (fluorometric), BioVision, Boai NKY Medical Holdings Ltd., China]. The 
compounds were dissolved in DMSO and added to the assay in at least 8 concentrations ranging from 1000 to $7.81 \mu \mathrm{mol} \mathrm{L} \mathrm{L}^{-1}$. Recombinant human aromatase stock was prepared by reconstitution with $1 \mathrm{~mL}$ of aromatase assay buffer. The content was mixed thoroughly by vortexing to obtain a homogeneous solution which was transferred to a 15-mL conical tube. The volume was brought to $2450 \mu \mathrm{L}$ with aromatase assay buffer and $50 \mu \mathrm{L}$ of NADPH production system (100X) was added for a final total volume of $2.5 \mathrm{~mL}$. Letrozole (Biovision) was used as a positive inhibition control. For solvent control, a small aliquot of aromatase assay buffer containing the organic solvent used to dissolve the test compounds was used. Reaction wells containing test compounds and corresponding no inhibitor-containing controls (which may also serve as solvent controls), as well as a background control

$\mathrm{R}_{1}-\mathrm{N}=\mathrm{C}=\mathrm{S}+\mathrm{NH}_{2} \mathrm{NH}_{2} \times \mathrm{H}_{2} \mathrm{O} \stackrel{\mathrm{EtOH}}{\longrightarrow} \stackrel{\mathrm{R}_{1} \mathrm{NHCNHNH}_{2}}{\stackrel{\text { II }}{2 .} \mathrm{CS}_{2} / \mathrm{NaOH}_{2}} \longrightarrow$

$1 \mathrm{a}-\mathrm{j}$

$2 \mathrm{a}-\mathrm{j}$<smiles>[R]Nc1nnc(S)s1</smiles>

\begin{tabular}{cccccc}
\hline Compd. & $\mathrm{R}_{1}$ & $\mathrm{R}_{2}$ & Compd. & $\mathrm{R}_{1}$ & $\mathrm{R}_{2}$ \\
\hline $\mathbf{4 a}$ & Methyl & Methyl & $\mathbf{4 l}$ & Methyl & Ethyl \\
$\mathbf{4 b}$ & Ethyl & Methyl & $\mathbf{4} \mathbf{m}$ & Ethyl & Ethyl \\
$\mathbf{4} \mathbf{c}$ & Methoxyethyl & Methyl & $\mathbf{4 n}$ & Methoxyethyl & Ethyl \\
$\mathbf{4} \mathbf{d}$ & Propyl & Methyl & $\mathbf{4 o}$ & Propyl & Ethyl \\
$\mathbf{4} \mathbf{e}$ & Isopropyl & Methyl & $\mathbf{4} \mathbf{p}$ & Isopropyl & Ethyl \\
$\mathbf{4 f}$ & Allyl & Methyl & $\mathbf{4 r}$ & Allyl & Ethyl \\
$\mathbf{4 g}$ & Butyl & Methyl & $\mathbf{4 s}$ & Butyl & Ethyl \\
$\mathbf{4 h}$ & Isobutyl & Methyl & $\mathbf{4}$ & Isobutyl & Ethyl \\
$\mathbf{4} \mathbf{i}$ & Cyclohexyl & Methyl & $\mathbf{4} \mathbf{u}$ & Cyclohexyl & Ethyl \\
$\mathbf{4 j}$ & Phenyl & Methyl & $\mathbf{4 v}$ & Phenyl & Ethyl \\
$\mathbf{4 k}$ & 4-Methylphenyl & Methyl & $\mathbf{4 y}$ & 4-Methylphenyl & Ethyl \\
\hline
\end{tabular}

Scheme 1. 
U. A. Çevik et al.: Synthesis and biological evaluation of novel 1,3,4-thiadiazole derivatives as possible anticancer agents, Acta Pharm. 70 (2020) 499-513.

(containing no fluorogenic aromatase substrate), were prepared. The plate was incubated for at least $10 \mathrm{~min}$ at $37^{\circ} \mathrm{C}$ to allow test ligands to interact with aromatase. After incubation, $30 \mu \mathrm{L}$ of the aromatase substrate/NADP ${ }^{+}$mixture was added to each well. Immediately (within $1 \mathrm{~min}$ ), the fluorescence at ex/em $=488 / 527 \mathrm{~nm}$ was measured.

\section{RESULTS AND DISCUSSION}

\section{Chemistry}

The compounds 4a-y were synthesized as outlined in Scheme 1. Firstly, suitable isothiocyanate and excess of hydrazine hydrate were reacted in order to obtain $N$-substituted hydrazinecarbothioamides (1a-j). Secondly, the reaction of compounds $\mathbf{1} \mathbf{a}-\mathbf{j}$ and carbondisulfide afforded 5-(substituted-amino)-1,3,4-thiadiazole-2-thiols (2a-j). The acetylation of thiadiazoles was performed using chloroacetyl chloride. Finally, 5-(substituted-amino)-1,3,4-thiadiazole-2-thiols (2a-j) and 2-chloro- $N$-(5-substituted-1,3,4-thiadiazol-2-yl)acetamides $(\mathbf{3} \mathbf{a}, \mathbf{b})$ were reacted in order to synthesize target compounds $\mathbf{4 a} \mathbf{a} \mathbf{y}$.

Stretching absorption of NH groups was observed at $3285-3303 \mathrm{~cm}^{-1}$ as expected. Carbonyl $(\mathrm{C}=\mathrm{O})$ group gave characteristic stretching absorption in the region of 1652-1666 $\mathrm{cm}^{-1}$. The stretching absorption at about $2916-2995 \mathrm{~cm}^{-1}$ was recorded for an aliphatic C-H bond. The stretching absorption belonging to 1,4-disubstituted benzene was detected at 844-850 $\mathrm{cm}^{-1}$ for compounds 4k (N-(5-methyl-1,3,4-thiadiazol-2-yl)-2-((5-( $p$-tolylamino)-1,3,4-thiadiazol-2-yl)thio)acetamide) and 4y (N-(5-ethyl-1,3,4-thiadiazol-2-yl)-2-((5- $p$ -tolylamino)-1,3,4-thiadiazol-2-yl)thio)acetamide).

In the ${ }^{1} \mathrm{H}$ NMR spectrum, the protons of the methyl $\left(-\mathrm{CH}_{3}\right)$ substituents were observed as a singlet peak between $2.60-2.62 \mathrm{ppm}$ for compounds $4 \mathbf{a}-\mathbf{k}$. The protons of the ethyl $\left(-\mathrm{C}_{2} \mathrm{H}_{5}\right)$ were observed as a triplet $\left(\mathrm{CH}_{3}\right)$ and quartet $\left(-\mathrm{CH}_{2}\right)$ peak between $1.28-1.33$ and 2.98-2.99 ppm, resp., for compounds 41-y. Methyl protons of compound $4 \mathbf{y}$ ( $N$-(5-ethyl-1,3,4-thiadiazol-2-yl)-2-((5-( $p$-tolylamino)-1,3,4-thiadiazol-2-yl)thio)acetamide) were found at $2.24 \mathrm{ppm}$ as a singlet. The protons of the ethyl group in compound $4 \mathbf{y}$ were observed at $1.28 \mathrm{ppm}$ as a triplet and $2.98 \mathrm{ppm}$ as a quartet. Moreover, methylene protons of compound $4 \mathbf{y}$ had singlet peak at $4.21 \mathrm{ppm}$. Disubstituted benzene protons of compound $4 \mathbf{y}$ had doublet peaks at $7.13 \mathrm{ppm}(J=8.3 \mathrm{~Hz})$ and $7.43 \mathrm{ppm}(J=8.5 \mathrm{~Hz})$. In the ${ }^{13} \mathrm{C}$ NMR spectrum, aliphatic peaks belonging to substituents were observed between $11.81 \mathrm{ppm}$ and $70.38 \mathrm{ppm}$. Aromatic carbons were identified between 116.72 and 175.17 ppm. Carbonyl carbon gave a peak between 166.82 and $175.17 \mathrm{ppm}$. The HRMS spectra of compounds were found to be in full agreement with their molecular formula.

\section{Cytotoxicity assay}

As shown in Table III, compounds $4 \mathbf{j}$ (N-(5-methyl-1,3,4-thiadiazol-2-yl)-2-((5-(phenylamino)-1,3,4-thiadiazol-2-yl)thio)acetamide), 4v (N-(5-ethyl-1,3,4-thiadiazol-2-yl)-2-((5-(phenylamino)-1,3,4-thiadiazol-2-yl)thio)acetamide) and 4y (N-(5-ethyl-1,3,4-thiadiazol-2-yl)-2-((5-(p-tolylamino)-1,3,4-thiadiazol-2-yl)thio)acetamide) were identified as the most active compounds. These compounds were tested against noncancer NIH3T3 cells, in order to measure the selectivity towards cancer cells. The $I C_{50}$ values of the compounds against cell lines are presented in Table III. 


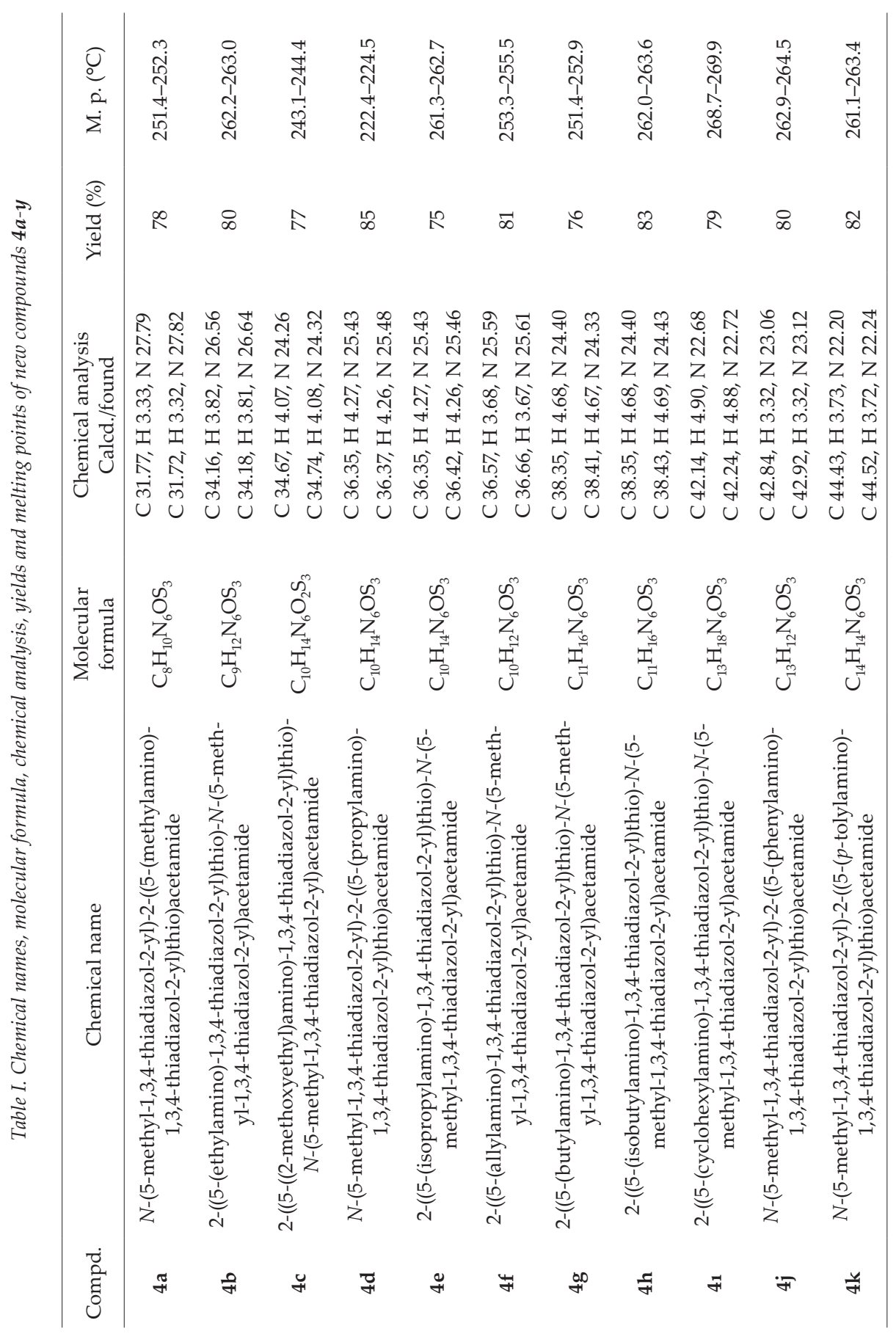




\begin{tabular}{|c|c|c|c|c|c|c|c|c|c|c|c|}
\hline $\begin{array}{l}0 \\
0 \\
\dot{0} \\
\dot{\Sigma}\end{array}$ & 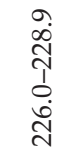 & 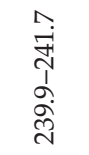 & 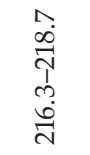 & $\begin{array}{l}\infty \\
\stackrel{\infty}{+} \\
\hat{N} \\
\grave{1} \\
\ddot{D} \\
\ddot{N}\end{array}$ & 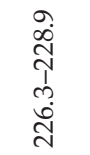 & 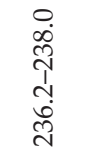 & 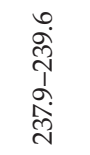 & 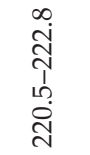 & 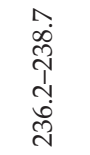 & 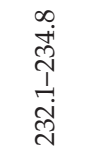 & 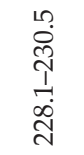 \\
\hline 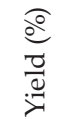 & $\hat{\Lambda}$ & $\stackrel{\circ}{\circ}$ & $\bar{\infty}$ & $\stackrel{10}{\infty}$ & $\stackrel{\infty}{\wedge}$ & ఉ & $\hat{\Sigma}$ & $\stackrel{10}{\infty}$ & న & $\stackrel{2}{1}$ & $\mathbb{N}$ \\
\hline 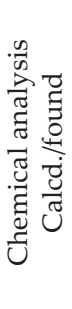 & 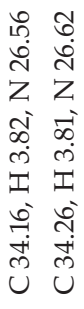 & 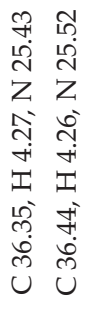 & 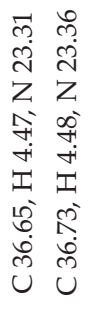 & 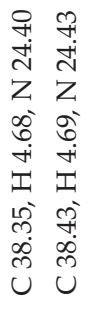 & 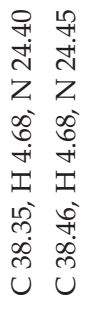 & 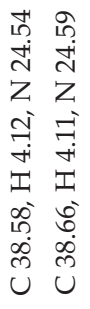 & 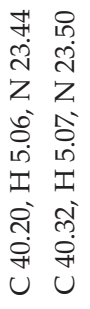 & 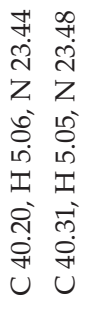 & 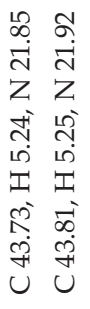 & 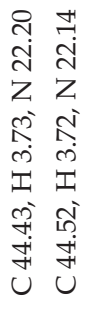 & 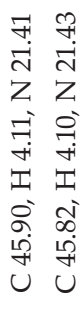 \\
\hline 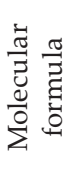 & 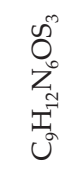 & 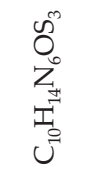 & $\begin{array}{l}\tilde{w}_{0}^{\infty} \\
O_{0} \\
Z_{0} \\
\vec{v} \\
\vec{v}\end{array}$ & $\begin{array}{l}\mathscr{D}_{0}^{m} \\
Z_{0} \\
\bar{F}^{=} \\
\vec{v}\end{array}$ & $\begin{array}{l}\tilde{O}^{m} \\
Z_{0} \\
Z^{0} \\
\vec{v}\end{array}$ & 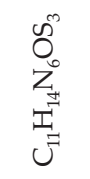 & 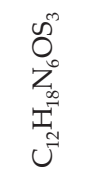 & $\begin{array}{l}\tilde{D}^{\infty} \\
Z_{0} \\
U^{\infty} \\
U^{\prime}\end{array}$ & 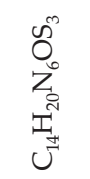 & 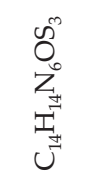 & 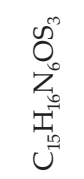 \\
\hline 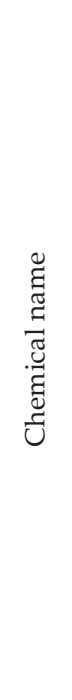 & 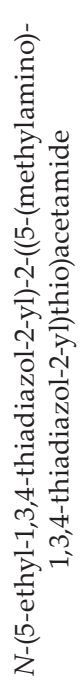 & 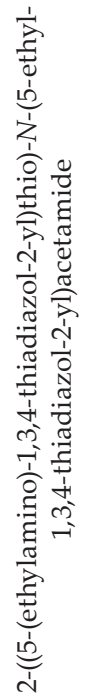 & 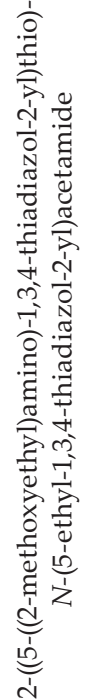 & 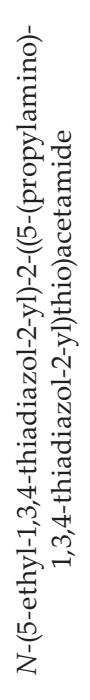 & 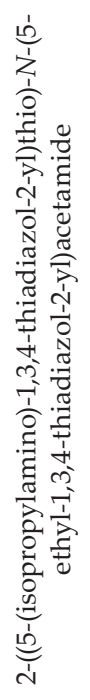 & 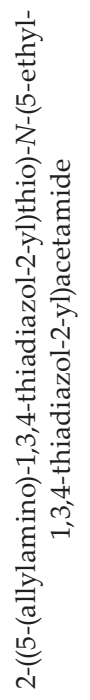 & 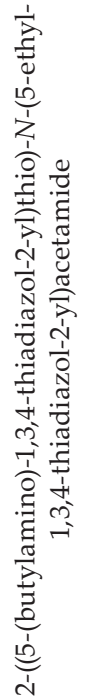 & 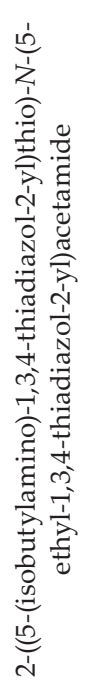 & 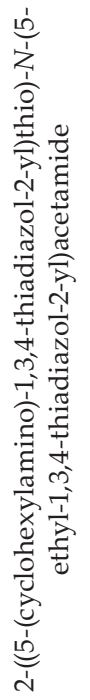 & 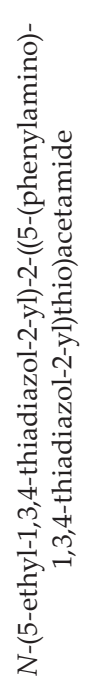 & 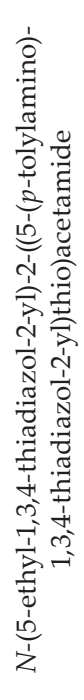 \\
\hline 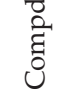 & $F$ & 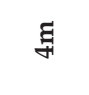 & $\mathscr{f}$ & \& & f & F & \& & f & 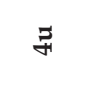 & $\dot{z}$ & 栗 \\
\hline
\end{tabular}




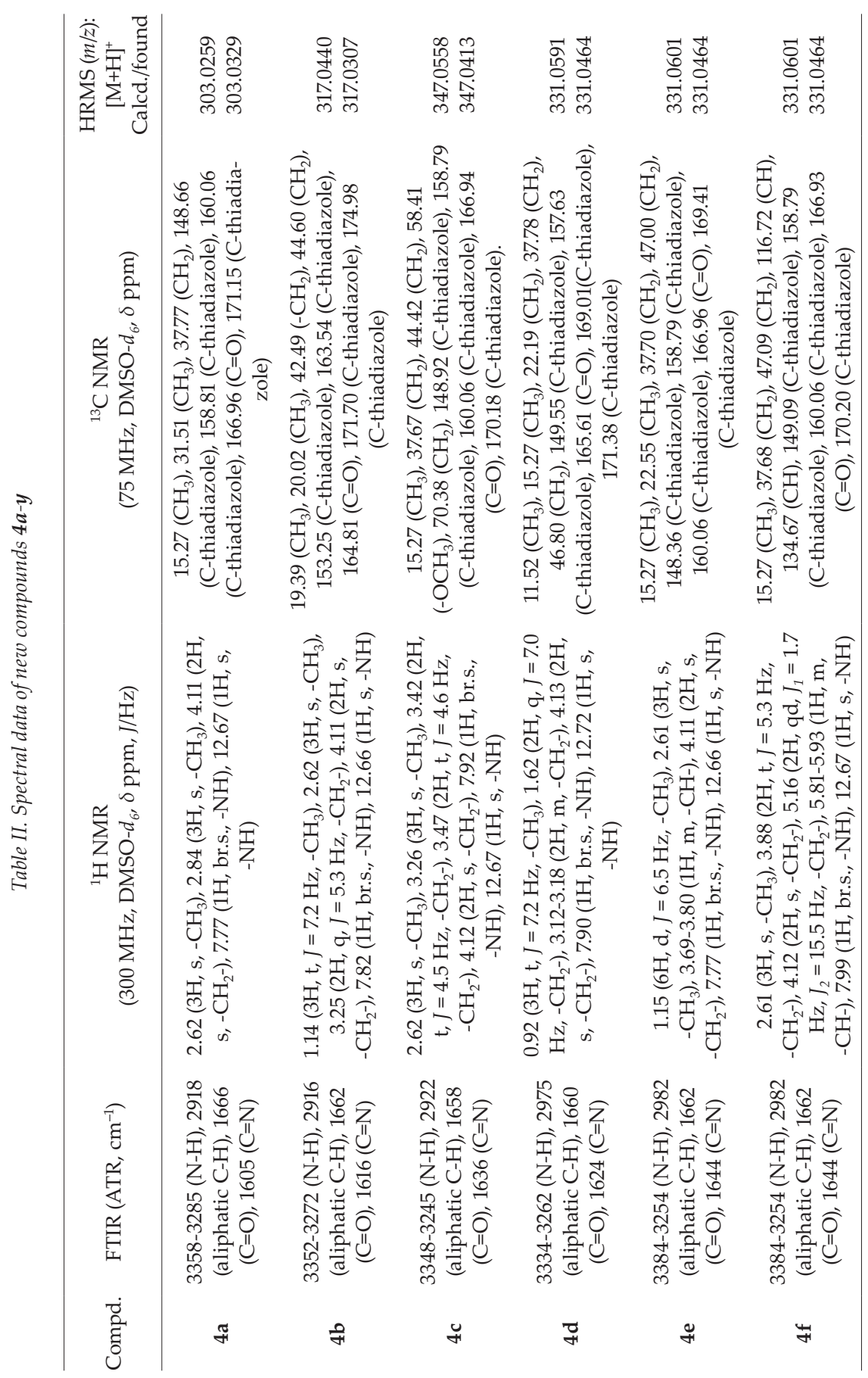




\begin{tabular}{|c|c|c|c|c|c|c|}
\hline 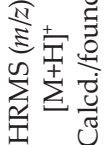 & 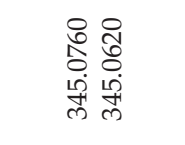 & 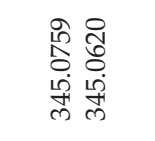 & 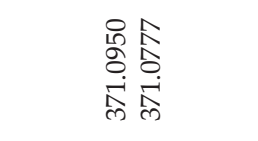 & 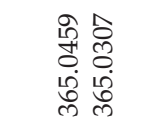 & 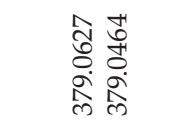 & 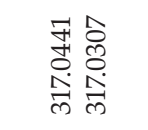 \\
\hline 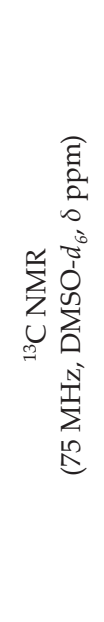 & 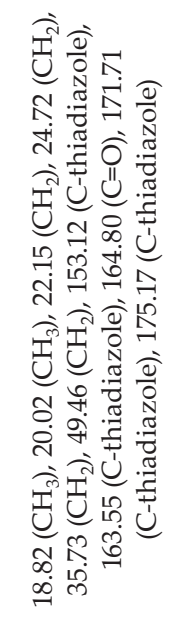 & 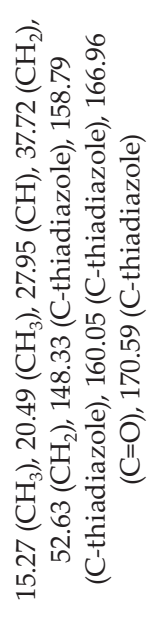 & 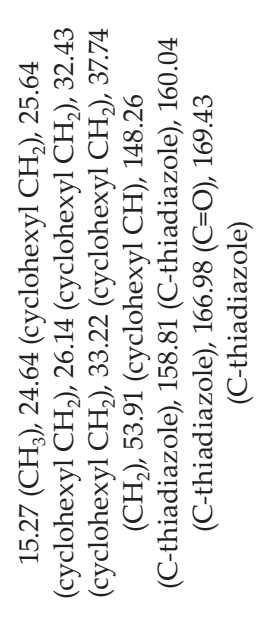 & 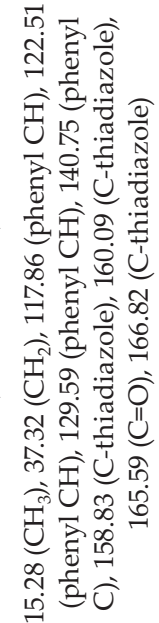 & 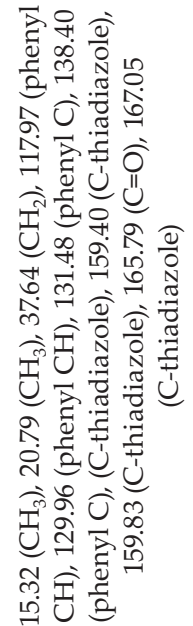 & 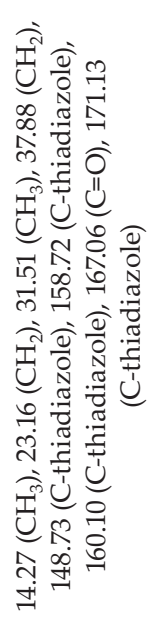 \\
\hline 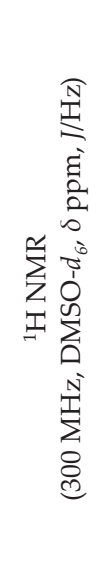 & 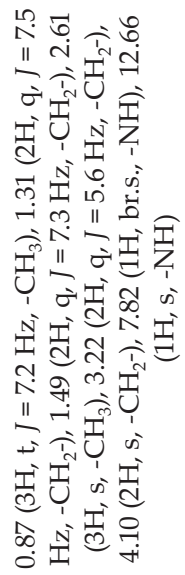 & 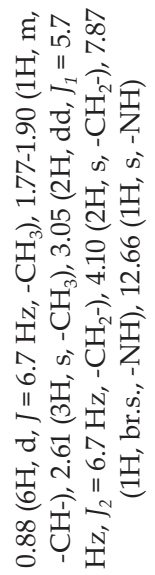 & 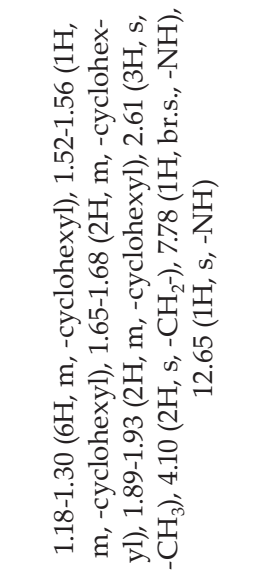 & 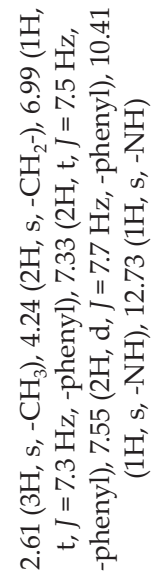 & 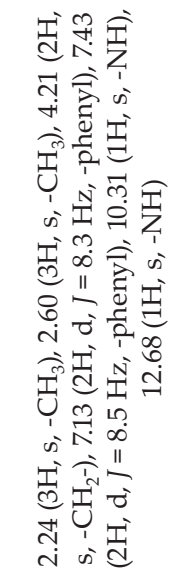 & 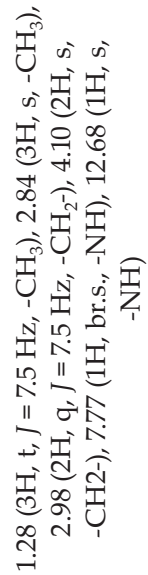 \\
\hline 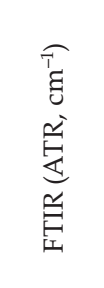 & 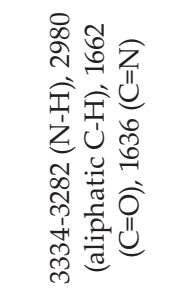 & 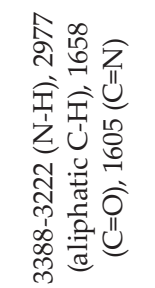 & 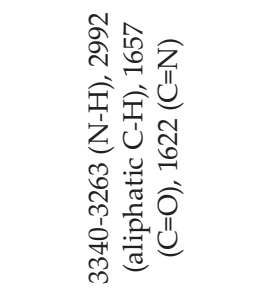 & 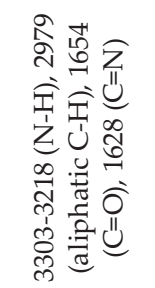 & 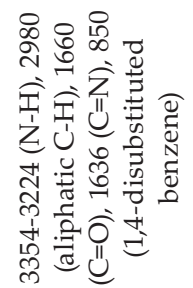 & 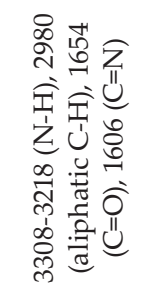 \\
\hline 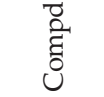 & $\stackrel{50}{a 0}$ & F & $F$ & F & F & $\overline{7}$ \\
\hline
\end{tabular}




\begin{tabular}{|c|c|c|c|c|c|c|}
\hline 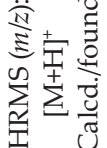 & 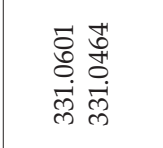 & 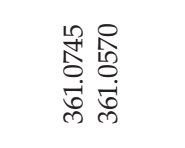 & 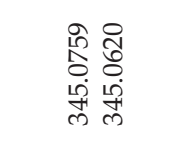 & 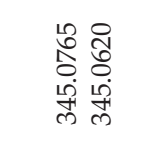 & 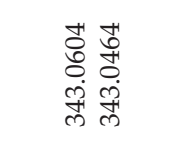 & 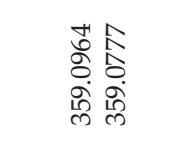 \\
\hline 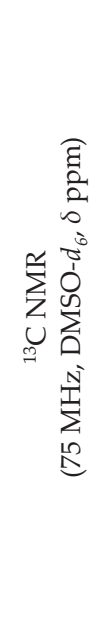 & 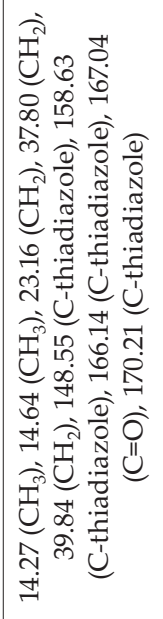 & 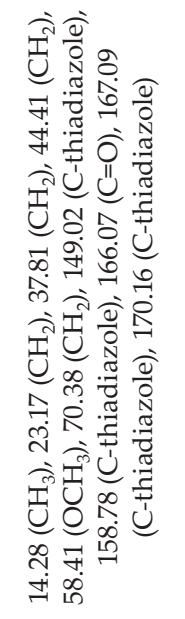 & 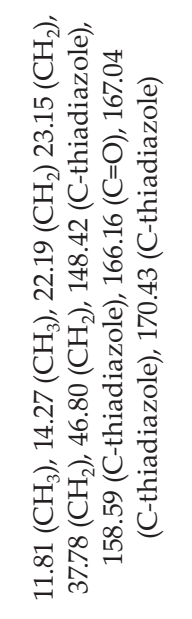 & 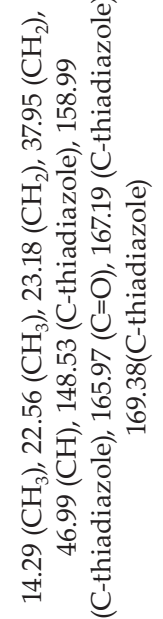 & 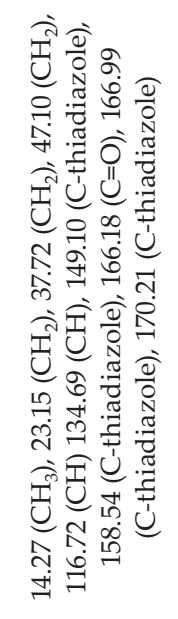 & 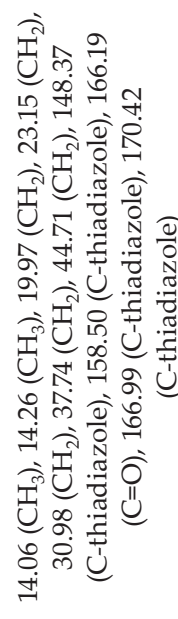 \\
\hline 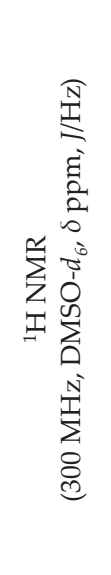 & 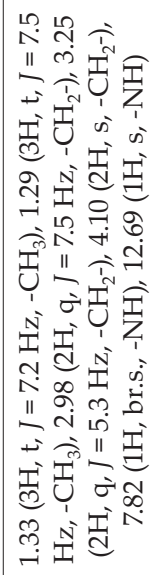 & 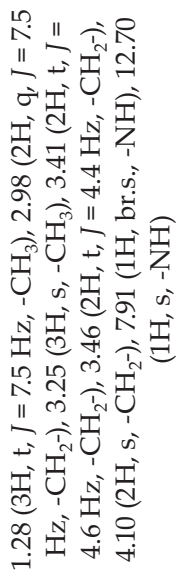 & 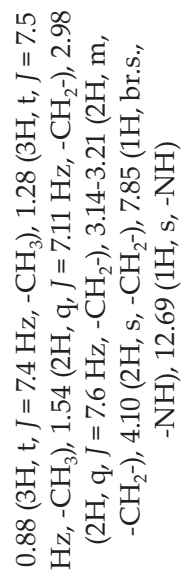 & 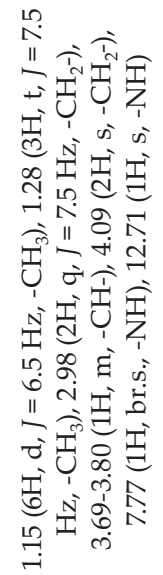 & 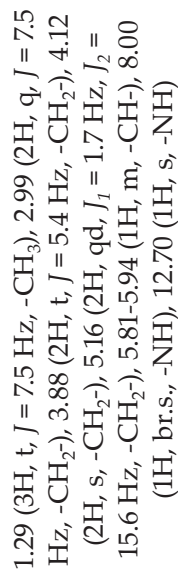 & 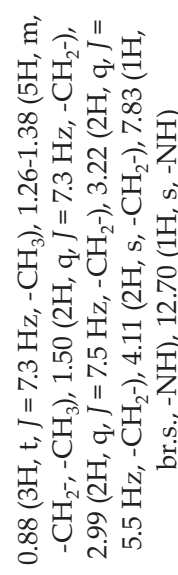 \\
\hline 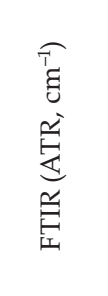 & 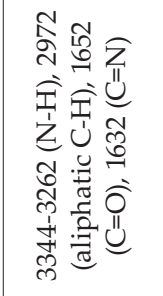 & 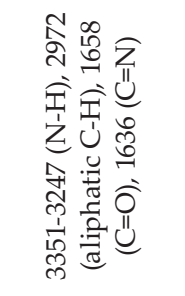 & 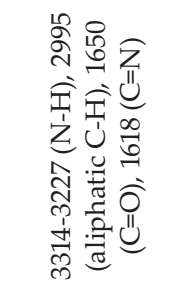 & 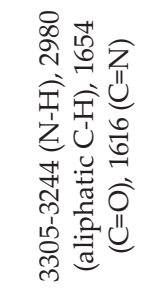 & 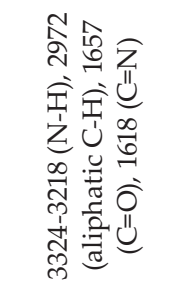 & 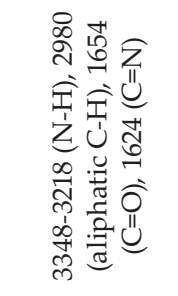 \\
\hline & 孚 & F & $f$ & & & \\
\hline
\end{tabular}




\begin{tabular}{|c|c|c|c|c|}
\hline$\sum_{n=1}^{5} \sum^{ \pm}$ & 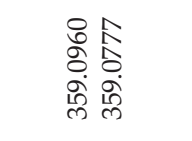 & 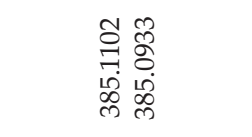 & 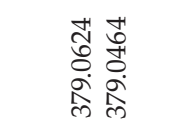 & 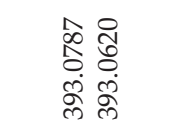 \\
\hline 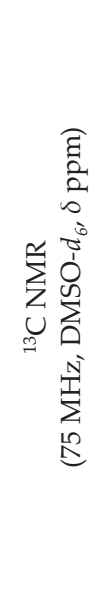 & 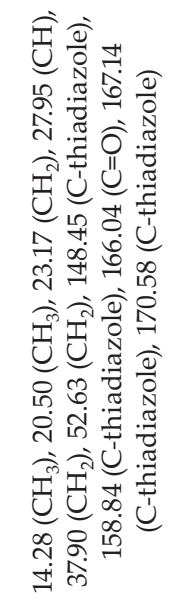 & 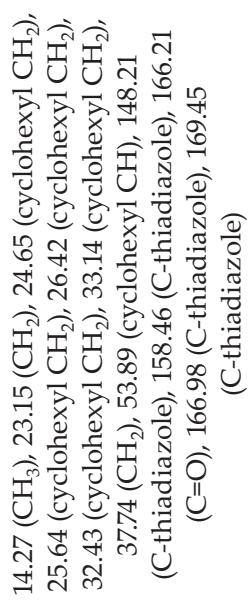 & 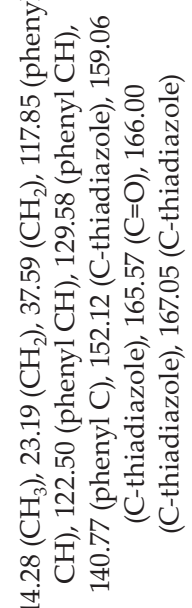 & 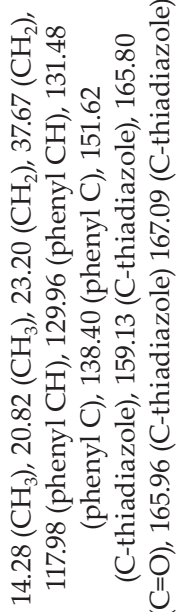 \\
\hline 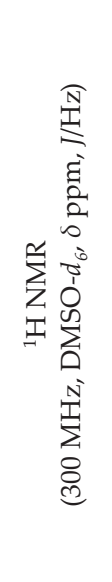 & 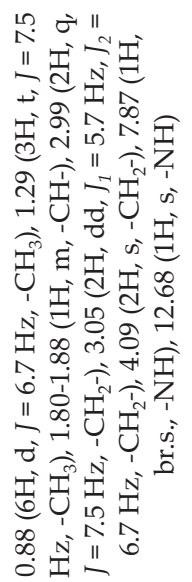 & 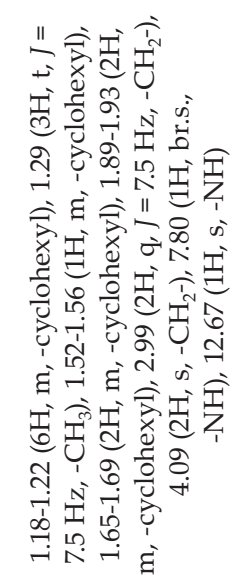 & 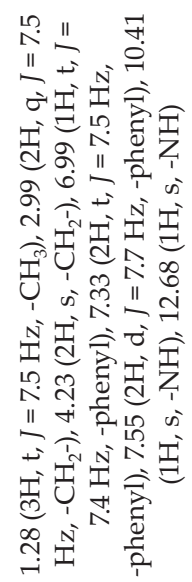 & 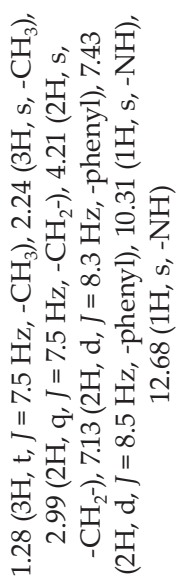 \\
\hline 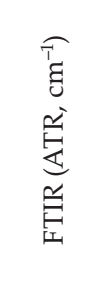 & 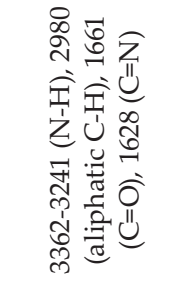 & 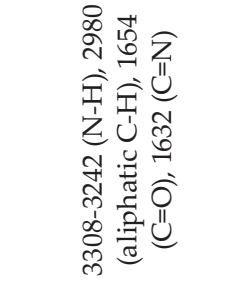 & 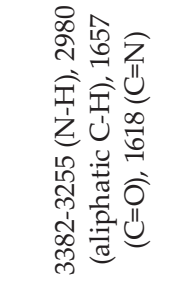 & 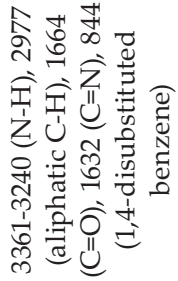 \\
\hline & $F$ & F & $f$ & \\
\hline
\end{tabular}


U. A. Çevik et al.: Synthesis and biological evaluation of novel 1,3,4-thiadiazole derivatives as possible anticancer agents, Acta Pharm. 70 (2020) 499-513.

Table III. IC ${ }_{50}$ values of compounds $4 \boldsymbol{a}$-y against cancer cell lines

\begin{tabular}{|c|c|c|c|c|c|}
\hline \multicolumn{6}{|c|}{$I C_{50}\left(\mathrm{mmol} \mathrm{L}^{-1}\right)$} \\
\hline Compd. & MCF-7 & A549 & Compd. & MCF-7 & A549 \\
\hline $4 a$ & $0.354 \pm 0.261$ & $0.119 \pm 0.071$ & $4 \mathrm{~m}$ & $0.806 \pm 0.246$ & $0.380 \pm 0.003$ \\
\hline $4 b$ & $\geq 1$ & $0.317 \pm 0.093$ & $4 n$ & $\geq 1$ & $0.318 \pm 0.062$ \\
\hline $4 c$ & $0.593 \pm 0.073$ & $0.848 \pm 0.124$ & 40 & $\geq 1$ & $\geq 1$ \\
\hline $4 d$ & $\geq 1$ & $\geq 1$ & $4 p$ & $\geq 1$ & $0.276 \pm 0.081$ \\
\hline $4 e$ & $\geq 1$ & $0.356 \pm 0.220$ & $4 r$ & $0.174 \pm 0.065$ & $0.223 \pm 0.086$ \\
\hline $4 \mathrm{f}$ & $\geq 1$ & $0.230 \pm 0.052$ & $4 \mathrm{~s}$ & $\geq 1$ & $0.694 \pm 0.068$ \\
\hline $4 \mathrm{~g}$ & $\geq 1$ & $\geq 1$ & $4 t$ & $0.133 \pm 0.019$ & $0.103 \pm 0.008$ \\
\hline $4 \mathrm{~h}$ & $\geq 1$ & $0.197 \pm 0.153$ & $4 u$ & $\geq 1$ & $\geq 1$ \\
\hline $4 i$ & $\geq 1$ & $\geq 1$ & $4 v$ & $0.116 \pm 0.041$ & $0.075 \pm 0.031$ \\
\hline $4 j$ & $\geq 1$ & $0.091 \pm 0.025$ & $4 y$ & $0.084 \pm 0.020$ & $0.034 \pm 0.008$ \\
\hline $4 \mathrm{k}$ & $\geq 1$ & $0.324 \pm 0.011$ & Cisplatin & $0.019 \pm 0.009$ & $0.013 \pm 0.003$ \\
\hline 41 & $\geq 1$ & $0.326 \pm 0.072$ & & & \\
\hline
\end{tabular}

Mean \pm SD, $n \geq 3$.

Table IV. IC ${ }_{50}$ against NIH3T3 and SI values of compounds $\mathbf{4 j}, \mathbf{4 v}$ and $\mathbf{4 y}$

\begin{tabular}{ccc}
\hline Compd. & $I_{50}\left(\mathrm{mmol} \mathrm{L}^{-1}\right)$ & SI \\
\hline $\mathbf{N} \mathbf{j}$ & $1.980 \pm 0.066$ & 21.76 \\
$\mathbf{4 v}$ & $1.764 \pm 0.077$ & 15.17 \\
$4 \mathbf{y}$ & $1.791 \pm 0.087$ & 21.31 \\
Cisplatin & $111.26 \pm 3.74_{5}$ & 8558.46 \\
\hline
\end{tabular}

Mean \pm SD, $n=3$.

\section{SAR studies}

Compound $4 \mathbf{j}$ and $4 \mathbf{v}$, both containing phenyl moiety, showed $I C_{50}$ values of 0.091 and $0.075 \mathrm{mmol} \mathrm{L}^{-1}$, resp., against A549, compared to the reference drug cisplatin $(0.013 \mathrm{mmol}$ $\mathrm{L}^{-1}$ ). Additionally, compound $4 \mathrm{y}$, containing toluene moiety, showed $I C_{50}$ values of 0.084 and $0.034 \mathrm{mmol} \mathrm{L}^{-1}$ against MCF-7 and A549, resp.

When the activity results are compared, it is seen that the introduction of an aromatic ring at the amine end of the thiadiazole increased the anticancer activity. However, the 
introduction of propyl or cyclohexyl at the amine end of the thiadiazole dramatically reduced the anticancer activity.

It is crucial that an anticancer agent affects the cancer cell line with having minimal or no effect on healthy cells. For this purpose, the cytotoxic effects of the most active compounds $(\mathbf{4} \mathbf{j}, \mathbf{4} \mathbf{v}$ and $\mathbf{4 y})$ were investigated on the NIH3T3 cell line. As seen in Table III, $I C_{50}$ of $0.084 \pm 0.020 \mathrm{mmol} \mathrm{L}^{-1}$ of compound $4 \mathbf{y}$ against MCF-7 was 20-times lower than its $I C_{50}$ of $1.790 \pm 8.706 \mathrm{mmol} \mathrm{L}^{-1}$ against NIH3T3 cell line. The $I C_{50}$ value of cisplatin was found to be greater than $1 \mathrm{mmol} \mathrm{L}^{-1}$ against NIH3T3 cell line.

The selectivity index (SI) was calculated for the effectiveness of selected compounds $4 \mathbf{j}, 4 \mathbf{v}$ and $4 \mathbf{y}$. The compounds with SI value $>3$ are considered to exhibit selective inhibition towards cancer cells (26). Compounds $4 \mathbf{j}, \mathbf{4} \mathbf{v}$ and $4 \mathbf{y}$ exhibited good selectivity since their SI values were in the range from 15 to 21 (Table IV).

\section{Aromatase inhibition assay}

Compound $4 \mathbf{y}$ which was found cytotoxic to MCF7 cancer cell line was submitted to aromatase inhibition assay. For this assay, a commercially available CYP19A inhibitor screening kit was used and letrozole was used as a positive control. Compound $4 \mathbf{y}$ caused $50 \%$ aromatase enzyme inhibition at $0.062 \pm 0.004 \mathrm{mmol} \mathrm{L}^{-1}$, whereas the respective value for letrozole was calculated as $0.020 \pm 0.001 \mathrm{mmol} \mathrm{L}^{-1}$.

\section{CONCLUSIONS}

In this study, we synthesized new $N$-(5-substituted-1,3,4-thiadiazol-2-yl)-2-[(5 (substituted-amino)-1,3,4-thiadiazol-2-yl)thio]acetamides (4a-y) and evaluated their anticancer potency. It was found that compound $4 \mathbf{y}$ ( $N$-(5-ethyl-1,3,4-thiadiazol-2-yl)-2-((5-( $p$-tolylamino)-1,3,4thiadiazol-2-yl)thio)acetamide) showed promising activity against A549 and MCF-7 cell lines as well as promising anti-aromatase activity.

Acknowledgements. - This study was financially supported by Anadolu University Scientific Research Projects Commission, Project no. 1906 S123.

Supplementary material available upon request.

\section{REFERENCES}

1. S. H. Giordano, D. S. Cohen, A. U. Buzdar, G. Perkins and G. N. Hortobagyi, Breast carcinoma in men: a population-based study, Cancer: Interdisc. Int. J. Am. Cancer Soc. 101 (2004) 51-57; https://doi. org/10.1002/cncr.20312

2. L. W. C. Chow, A. Yun-San Yip, W. T. Y. Loo and M. Toi, Evaluation of neoadjuvant inhibition of aromatase activity and signal transduction in breast cancer, Cancer Lett. 262 (2008) 232-238; https:// doi.org/10.1016/j.canlet.2007.12.003

3. A. M. Farag, A. S. Mayhoub, T. M. Eldebss, A. G. E. Amr, K. A. Ali, N. A. Abdel-Hafez and M. M. Abdulla, Synthesis and structure-activity relationship studies of pyrazole-based heterocycles as antitumor agents, Arch. Pharm. 343 (2010) 384-396; https://doi.org/10.1002/ardp.200900176

4. Z. Sahin, M. Ertas, B. Berk, S.N. Biltekin, L. Yurttas and S. Demirayak, Studies on non-steroidal inhibitors of aromatase enzyme; 4-(aryl/heteroaryl)-2-(pyrimidin-2-yl) thiazole derivatives, Bioorg. Med. Chem. 26 (2018) 1986-1995; https://doi.org/10.1016/j.bmc.2018.02.048 
U. A. Çevik et al.: Synthesis and biological evaluation of novel 1,3,4-thiadiazole derivatives as possible anticancer agents, Acta Pharm. 70 (2020) 499-513.

5. R. J. Santen, E. Samojlik, A. Lipton, H. Harvey, E. B. Ruby, S. A. Wells and J. Kendall, Kinetic, hormonal and clinical studies with aminoglutethimide in breast cancer, Cancer 39 (1977) 2948-2958; https://doi.org/10.1002/1097-0142(197706)39:6<2948::AID-CN CR2820390681 >3.0.CO;2-9

6. R. J. Santen, S. Santner, B. Davis, J. Veldhuis, E. Samojlik and E. Ruby, Aminoglutethimide inhibits extraglandular estrogen production in postmenopausal women with breast carcinoma, J. Clin. Endocrinol. Metab. 47 (1978) 1257-1265; https://doi.org/10.1210/jcem-47-6-1257

7. M. Ertas, Z. Sahin, B. Berk, L. Yurttas, S.N. Biltekin and S. Demirayak, Pyridine-substituted thiazolylphenol derivatives: Synthesis, modeling studies, aromatase inhibition, and antiproliferative activity evaluation, Arch. Pharm. 351 (2018) 1700272; https://doi.org/10.1002/ardp.201700272

8. P. Furet, C. Batzl, A. Bhatnagar, E. Francotte, G. Rihs and M. Lang, Aromatase inhibitors: synthesis, biological activity, and binding mode of azole-type compounds, J. Med. Chem. 36 (1993) 13931400 .

9. C. D. Jones, M. A. Winter, K. S. Hirsch, N. Stamm, H. M. Taylor, H. E. Holden, J. D. Davenport, E. V. Krumkalns and R. G. Suhr, Estrogen synthetase inhibitors. 2. Comparison of the in vitro aromatase inhibitory activity for a variety of nitrogen heterocycles substituted with diarylmethane or diarylmethanol groups, J. Med. Chem. 33 (1990) 416-429.

10. A. Cavalli and M. Recanatini, Looking for selectivity among cytochrome P450s inhibitors, J. Med. Chem. 45 (2002) 251-254; https://doi.org/10.1021/jm015567k

11. M. L. Fascio, C. S. Sepúlveda, E. B. Damonte and N. B. D'Accorso, Synthesis and antiviral activity of some imidazo $[1,2-b][1,3,4]$ thiadiazole carbohydrate derivatives, Carbohydr. Res. 480 (2019) 6166; https://doi.org/10.1016/j.carres.2019.05.003

12. J. Chen, C. Yi, S. Wang, S. Wu, S. Li, D. Hu and B. Song, Novel amide derivatives containing 1,3,4-thiadiazole moiety: Design, synthesis, nematocidal and antibacterial activities, Bioorg. Med. Chem. Lett. 29 (2019) 1203-12010; https://doi.org/10.1016/j.bmcl.2019.03.017

13. G. Serban, Future prospects in the treatment of parasitic diseases: 2-Amino-1,3,4-thiadiazoles in leishmaniasis, Molecules 24 (2019) 1557-1579; https://doi.org/10.3390/molecules24081557

14. D. C. Sekhar, D. V. Rao, A. T. Rao, U. L. Kumar and A. Jha, Design and synthesis of 1,3,4-thiadiazole derivatives as novel anticancer and antitubercular agents, Russ. J. Gen. Chem. 89 (2019) 770779; https://doi.org/10.1134/S1070363219040224

15. A. Foroumadi, Z. Kargar, A. Sakhteman, Z. Sharifzadeh, R. Feyzmohammadi, M. Kazemi and A. Shafiee, Synthesis and antimycobacterial activity of some alkyl [5-(nitroaryl)-1,3,4-thiadiazol2-ylthio] propionates, Bioorg. Med. Chem. Lett. 16 (2006) 1164-1167; https://doi.org/10.1016/j. bmcl.2005.11.087

16. B. Sharma, A. Verma, S. Prajapati and U. K. Sharma, Synthetic methods, chemistry, and the anticonvulsant activity of thiadiazoles, Int. J. Med. Chem. 2013 (2013) Article ID 348948 (16 pages); https:// doi.org/10.1155/2013/348948

17. V. Mathew, J. Keshavayya, V. P. Vaidya and D. Giles, Studies on synthesis and pharmacological activities of 3, 6-disubstituted-1,2,4-triazolo [3,4-b]-1,3,4-thiadiazoles and their dihydro analogues, Eur. J. Med. Chem. 42 (2007) 823-840; https://doi.org/10.1016/j.ejmech.2006.12.010

18. İ. Celik, G. Ayhan-Kılcıgil, B. Guven, Z. Kara, A. S. Gurkan-Alp, A. Karayel and A. Onay-Besikci, Design, synthesis and docking studies of benzimidazole derivatives as potential EGFR inhibitors, Eur. J. Med. Chem. 173 (2019) 240-249; https://doi.org/10.1016/j.ejmech.2019.04.012

19. M. F. Abo-Ashour, W. M. Eldehna, A. Nocentini, H. S. Ibrahim, S. Bua, H. A. Abdel-Aziz, S. M. Abou-Seri and C. T. Supuran, Novel synthesized SLC-0111 thiazole and thiadiazole analogues: Determination of their carbonic anhydrase inhibitory activity and molecular modeling studies, Bioorg. Chem. 87 (2019) 794-802; https://doi.org/10.1016/j.bioorg.2019.04.002

20. D. I. Pérez, M. Pistolozzi, V. Palomo, M. Redondo, C. Fortugno, C. Gil, G. Felix, A. Martinez and C. Bertucci, 5-Imino-1,2,4-thiadiazoles and quinazolines derivatives as glycogen synthase kinase 
$3 \beta$ (GSK-3 $\beta$ ) and phosphodiesterase 7 (PDE7) inhibitors: determination of blood-brain barrier penetration and binding to human serum albumin, Eur. J. Pharm. Sci. 45 (2012) 677-684; https://doi. org/10.1016/j.ejps.2012.01.007

21. Y. Bahmani, T. Bahrami and A. Alabadi, Synthesis, cytotoxicity assessment and molecular docking of $\mathrm{n}$-(5-(substituted-benzylthio)-1,3,4-thiadiazole-2-yl)-2- $p$-fluorophenylacetamide derivatives as tyrosine kinase inhibitors, Indian J. Pharm. Sci. 81 (2019) 63-70.

22. A. S. Mayhoub, L. Marler, T. P. Kondratyuk, E. J. Park, J. M. Pezzuto and M. Cushman, Optimizing thiadiazole analogues of resveratrol versus three chemopreventive targets, Bioorg. Med. Chem. 20 (2012) 510-520; https://doi.org/10.1016/j.bmc.2011.09.031

23. M. Varache-Lembege, S. Moreau, S. Larrouture, D. Montaudon, J. Robert and A. Nuhrich, Synthesis and antiproliferative activity of aryl- and heteroaryl-hydrazones derived from xanthone carbaldehydes, Eur. J. Med. Chem. 43 (2008) 1336-1343; https://doi.org/10.1016/j.ejmech.2007.09.003

24. M. V. Berridge, P. M. Herst and A. S. Tan, Tetrazolium dyes as tools in cell biology: new insights into their cellular reduction, Biotechnol. Annu. Rev. 11 (2005) 127-152; https://doi.org/10.1016/S13872656(05)11004-7

25. Ü. Demir Özkay, Ö. D. Can, B. N. Sağlık, U. Acar Çevik, S. Levent, Y. Özkay, S. Ilgın and Ö. Atlı, Design, synthesis, and AChE inhibitory activity of new benzothiazole-piperazines, Bioorg. Med. Chem. Lett. 26 (2016) 5387-5394; https://doi.org/10.1016/j.bmcl.2016.10.041

26. M. R. Haider, K. Ahmad, N. Siddiqui, Z. Ali, M. J. Akhtar, N. Fuloria, S. Fuloria, M. Ravichandran and M. S. Yar, Novel 9-(2-(1-arylethylidene)hydrazinyl)acridine derivatives: Target topoisomerase 1 and growth inhibition of HeLa cancer cells, Bioorg. Chem. 88 (2019) Article ID 102962; https://doi. org/10.1016/j.bioorg.2019.102962 